\title{
Estigma, discriminación y concepto de enfermedad mental.
}

\author{
Stigma, discrimination and concept of mental illness. \\ Santiago Stucchi-Portocarrero ${ }^{1, a}$

\section{RESUMEN}

El estigma y la discriminación contra los enfermos mentales han existido a lo largo de toda la historia, pese a los cambios conceptuales de la enfermedad mental, desde las explicaciones sobrenaturales hasta las de naturaleza científico-racional. Se presentan cinco episodios históricos representativos de abuso o persecución contra personas con trastornos mentales, cada uno respondiendo a modelos conceptuales diferentes: la caza de brujas, el encierro, la degeneración y la eugenesia, la lobotomía, y los hospitales-cárceles soviéticos.

PALABRAS CLAVE: estigma, discriminación, trastorno mental, psiquiatría.

\section{SUMMARY}

Stigma and discrimination against mentally ill people have existed throughout history, despite the conceptual changes of mental illness, from the supernatural explications to those of scientific-rational nature. We present five representative historical episodes of abuse or persecution of people with mental disorders, each responding to different conceptual models: the witch hunt, the confinement, degeneration and eugenics, lobotomy, and soviet hospitals-prisons.

KEY WORDS: Stigma, discrimination, mental disorder, psychiatry.

\section{INTRODUCCIÓN}

Durante la mayor parte de la antigüedad se atribuyó a los dioses y otros seres sobrenaturales la génesis de todos los eventos inexplicables, sean favorables o desafortunados, incluyendo entre éstos a las enfermedades. Por ejemplo, en la Biblia podemos leer las siguientes consecuencias de la desobediencia: "Jehová te herirá con la úlcera de Egipto, con tumores, con sarna, y con comezón de que no puedas ser curado. Jehová te herirá con locura, ceguera y turbación de espíritu"(1). El hombre proyectó así en los seres sobrenaturales sus propias vivencias subjetivas, animando la naturaleza con intencionalidades muy humanas más no muy divinas. En ocasiones la dolencia no tenía un origen punitivo, sino más bien un propósito revelador. Como hizo decir Platón a Sócrates en Fedro: "Esto sería muy bueno, si fuese evidente que el delirio es un mal; pero es todo lo contrario; al delirio inspirado por los dioses es al que somos deudores de los más grandes bienes" (2).

Con el paso de los siglos empezaron a plantearse hipótesis más terrenales para explicar las enfermedades. Así, en el siglo IV a.C. Hipócrates comentó lo siguiente sobre la epilepsia: "No me parece que sea en nada más divina que las demás enfermedades, ni más sagrada, sino que tiene también una causa natural (...) A mi parecer, aquellos que por primera vez hicieron sagrada esta afección eran lo mismo que los actuales magos y purificadores, vagabundos impostores y charlatanes; éstos pretenden

1 Instituto Nacional de Salud Mental "Honorio Delgado - Hideyo Noguchi”. Facultad de Medicina Alberto Hurtado, Universidad Peruana Cayetano Heredia. Lima, Perú.

a Médico psiquiatra. 
ser de vehemente piedad y saber más; pero utilizan lo divino para ocultar su impotencia y desconcierto por no contar con ninguna ayuda que ofrecer" (3). La concepción científico-racional de las enfermedades mentales lograría paulatinamente compartir su lugar con las explicaciones mágico-religiosas hasta llegar a un predominio sobre las mismas, luego de sobrevivir a un pronunciado debilitamiento medieval.

Sin embargo, aunque el concepto científicoracional ha permitido una mejor comprensión de los fenómenos psicopatológicos, así como un innegable desarrollo terapéutico durante la última centuria, su preeminencia no logró extinguir el estigma ni la discriminación contra el enfermo mental. Por otro lado, la actividad médica científica no siempre se ha ejercido de manera impoluta, sino que muchas veces se ha visto tergiversada por ideologías sociales y políticas de diversa índole. De este modo, el estigma ha persistido cambiando únicamente de disfraz: los poseídos medievales pasaron a ser sucesivamente incurables, degenerados o disidentes políticos. Víctimas todos ellos de discriminación y en muchos casos de abusos o franca persecución y exterminio, llevados a cabo bajo la batuta de un supuesto conocimiento científico objetivo y desprejuiciado.

Se presentan a continuación cinco episodios históricos en los cuales conceptos muy distintos sobre la enfermedad mental se asemejaron al momento de propiciar actos negativos contra quienes la padecían:

\section{LA CAZA DE BRUJAS}

Durante la edad media predominó claramente el concepto mágico-religioso de la enfermedad mental, imponiéndose la idea de la posesión diabólica para intentar explicar algunos casos de comportamientos extraños y perturbadores. No obstante, la cura medieval de la posesión estaba dirigida por lo general a expulsar al demonio del cuerpo y no hacia el cuerpo mismo, siendo relativamente raros los actos de crueldad contra los supuestos poseídos. Inclusive en el Concilio de Paderborn (año 785) la Iglesia Católica condenó la creencia en brujas así como la persecución de las mismas, y mediante el Canon Episcopi (año 906) consideró los temas de la brujería como "enormes mentiras" (4). Tal situación fue cambiando desde finales de la edad media.

En 1484 el Papa Inocencio VIII promulgó la bula Summis desiderantes affectibus, derogando en la práctica el Canon Episcopi y propiciando la persecución de la hechicería (4). El Malleus Malleficarum, escrito por dos monjes dominicos y publicado en 1487, dio el marco teórico perfecto para la caza de brujas. La supuesta poseída -generalmente mujer y muchas veces enferma mental- no era más una pobre víctima, si no alguien que había optado voluntariamente por el mal, alguien en quien no había redención alguna, por lo cual debía liberarse el alma envilecida quemando el cuerpo corrupto. La hoguera devino así en acto purificador y misericordioso, y decenas de miles de supuestas brujas terminaron quemadas en la hoguera. El carácter "misógino y antierótico" (5) de estos hechos no fue casual; para los autores del Malleus, había motivos para que la mayor cantidad de brujas perteneciera al sexo femenino: "la razón natural es que (la mujer) es más carnal que el hombre, como resulta claro de sus muchas abominaciones carnales. Y debe señalarse que hubo un defecto en la formación de la primera mujer, ya que fue formada de una costilla curva, es decir, la costilla del pecho, que se encuentra encorvada, por decirlo así, en dirección contraria a la de un hombre. Y como debido a este defecto es un animal imperfecto, siempre engaña" (6). Los procesos por brujería no fueron patrimonio de la Iglesia Católica, siendo continuados con entusiasmo por los seguidores reformistas de Lutero y de Calvino. La tormenta amainó a partir del siglo XVIII, aunque no cesó del todo hasta principios del siglo XIX, calculándose en decenas de miles el total de víctimas. Tales fueron los resultados de una concepción sobrenatural del mundo llevada al extremo.

\section{EL ENCIERRO}

Según Foucault, en 1656 el rey Luis XIV decretó el encierro de todos los elementos marginales de la sociedad. Así "indigentes, bandoleros, vagos, prostitutas, menesterosos y, sobretodo, pordioseros, constituían la mayor parte de este ejército monstruoso de lo irracional; no obstante, sus dirigentes simbólicos eran los locos y los idiotas" (7). Aunque posteriormente se ha cuestionado la teoría foucaultiana de un encierro masivo y relativamente súbito de los locos como parte de una conspiración estatal contra la irracionalidad en aras de un orden social burgués, de todos modos a partir del siglo XVII se impuso una visión manicomial del trastorno mental, edificándose enormes establecimientos específicos para el confinamiento de todos los llamados insanos.

Pero si algún propósito humanitario pudo haber motivado la fundación de los hospitales generales, aquél se fue desvirtuando con el tiempo debido a las 
condiciones imperantes en el interior de los mismos. Foucault nos brinda la siguiente aterradora descripción de la Salpêtrière a fines del siglo XVIII: "Las locas atadas por excesos de furor son encadenadas como perros a la puerta de su cuarto, y separadas de los guardianes y de los visitantes por un largo corredor defendido por una verja de hierro; se les pasan entre los barrotes la comida y la paja, sobre la cual se acuestan; por medio de rastrillos se retira una parte de las suciedades que las rodean" (8). En el Perú, José Casimiro Ulloa informó lo siguiente en 1857 acerca de las loquerías de los hospitales San Andrés y Santa Ana: "Hemos recorrido esas especies de cárceles que en Lima se honra con el nombre de casa de locos, nuestro corazón ha sido cruelmente herido de pesadumbre y angustia. Al ver el semblante de estos desdichados recostados en inmundos colchones sobre el suelo, o sobre gruesas tarimas, encerrados a pares en estrechas y húmedas celdas, sin más mueble que las vasijas de barro indispensables a sus más apremiantes necesidades, al verlos atados a las paredes de ellas con cadenas de hierro, o colocados sus pies en un cepo, al mirarlos vagar por un corredor estrecho, sin otro cuadro a que volver los ojos que el espectáculo de las desgracias de sus compañeros de cárcel, no hemos podido alejar de nuestra memoria el recuerdo de las lastimosas escenas de que hemos hecho mención" (9). El viajero suizo Johann Jakob von Tschudi relató que "el 30 de noviembre, el día de San Andrés, se permite el acceso al público (al Hospital de San Andrés). Esta ocasión es aprovechada por los habitantes de Lima para poder divertirse mirando a los locos. Es un espectáculo escandaloso ver a estos infelices expuestos como objetos de burlas y de curiosidad del público. La colección de limosnas de los numerosos visitantes es el fin de esta costumbre, pero aun así es reprochable" (10).

A fines del siglo XVIII Philippe Pinel instauró el denominado tratamiento moral (aunque vale aclarar que Vincenzo Chiarugi se le había adelantado en Italia), dejando atrás las cadenas y los grilletes, que formaban parte habitual del trato a los internos. $\mathrm{La}$ tarea, sin embargo, no fue sencilla, y debió tropezar con cuestionamientos como el de Couthon, quien le espetó lo siguiente: “¡Caramba, ciudadano! ¿Es que tú mismo estás loco, para querer desencadenar a semejantes animales?" (8). Aunque llegó tarde al Perú, la reforma dio impulso a la fundación del Hospicio de la Misericordia en 1859, lugar donde fueron trasladados todos los enfermos mentales de San Andrés y SantaAna. Sin embargo, los alienistas del siglo XIX no buscaron desligarse del manicomio; por el contrario, centraron la cura del enfermo en el establecimiento mismo. "El encierro no era, en su concepto, el camino hacia la terapia, era la terapia misma" (11). Lamentablemente el encierro curativo pronto mostraría sus límites; pocos años después de inaugurado, el Hospicio de la Misericordia -más conocido como el Manicomio del Cercado- se encontraba hacinado y con pocos recuerdos de las buenas intenciones fundacionales. En 1918 debió mudar a sus internos al Asilo Colonia de la Magdalena (el actual Hospital Víctor Larco Herrera), el cual repetiría la triste historia de hacinamiento, maltrato y abandono estatal durante décadas. En su sincera vocación por incluir al mayor número posible de curables, el modelo manicomial llevaba en sí mismo los gérmenes de su autodestrucción (muy recomendable en este punto resulta la novela "El aliniesta”, de Joaquin María Machado de Assis, 2008).

\section{LA DEGENERACIÓN Y LA EUGENESIA}

En 1857 Bénedict Augustine Morel planteó que la degeneración es un proceso acumulativo e irreversible de desviación del tipo humano normal, que progresaba a través de sucesivas generaciones hasta terminar en la extinción; de este modo, la historia familiar puede revelar una decadencia que va desde la neurosis, pasando por el alcoholismo, la prostitución, la criminalidad y la psicosis, hasta llegar a la imbecilidad y esterilidad. El individuo degenerado podía serlo en todos los aspectos imaginables; la descripción de Debay es elocuente: "Su físico es enfermizo, su inteligencia muy poco por encima de la bestia: la mayor parte son sordos o mudos y pasan su vida en la pereza y en la imbecilidad. (...) de limitada inteligencia, malos y traidores, pero debemos añadir que son, además, lascivos como los monos, y no sería prudente dejar a una joven sola con ellos" (12). La teoría de la degeneración alcanzó particular relevancia en la criminalística, mediante las ideas de Cesare Lombroso y su concepto del criminal nato, tipo degenerado con estigmas físicos que lo delatan, tales como prognatismo y frente estrecha; una "reminiscencia del hombre primitivo" (13). En el ámbito de la sexualidad, Richard von Krafft-Ebing tipificó las "perversiones sexuales" como "signos funcionales de degeneración", incluyendo en aquel vasto grupo la aparición infantil de la sexualidad, la reaparición senil de la sexualidad, el sadismo, el masoquismo, la satiriasis, el fetichismo y la homosexualidad, entre muchos otros (14).

El nihilismo terapéutico del degeneracionismo, así como la frontera zanjada entre el ser humano ideal y el degenerado, llevarían a la postre a desarrollar la 
Estigma, discriminación y concepto de enfermedad mental.

idea del mejoramiento de la especie humana. Nacía así la eugenesia, definida por Galton como "la ciencia que trata sobre todas las influencias que mejoran las cualidades innatas de una raza, y también sobre aquellas que las desarrollan hasta la mayor ventaja" (15). La nueva ciencia fue adoptada como política de gobierno por algunos países. Tal fue el caso de Estados Unidos, en donde a partir de 1896, diversos estados aprobaron leyes que prohibían el matrimonio a los "epilépticos, imbéciles o débiles mentales". En 1910 se fundó la Oficina de Registro de Eugenesia, y en 1927, la Corte Suprema de los Estados Unidos ratificó las leyes que prohibían el matrimonio entre enfermos mentales, y que además obligaban la esterilización de los mismos; se calcula que bajo estas leyes, 64 mil personas fueron esterilizadas forzosamente entre 1927 y 1963. En Suecia, el parlamento aprobó por unanimidad las leyes eugenésicas destinadas a preservar "la pureza de la raza nórdica", llevando a la esterilización forzada a casi 63 mil personas entre 1935 y 1975. En Alemania más de 400 mil individuos fueron esterilizados en el periodo inicial del Tercer Reich (1934-1937), bajo la Ley de Prevención de Enfermedades Hereditarias (16). Más adelante, la esterilización no fue suficiente, y el genocidio se convirtió en la "solución final" para millones de "indeseables", que ya no eran solamente los discapacitados mentales, sino también los judíos, gitanos y homosexuales. La concepción biológica del trastorno mental en su versión más pesimista no solo no había salvado a los enfermos, sino que había "degenerado" hasta propiciar su exterminio.

\section{LA LOBOTOMÍA}

En 1848 un un obrero norteamericano de nombre Phineas Gage fue víctima de una explosión, producto de la cual una barra de metal de $2,5 \mathrm{~cm}$. de diámetro atravesó su cráneo. Aunque Gage sobrevivió, su personalidad cambió por completo, tornándose "infantil, caprichoso, desconsiderado, irreverente y poco criterioso", no volviendo a tener nunca más un empleo estable, y muriendo en la indigencia 13 años después. John Harlow -el médico que lo atendió desde el principio- opinó que "el equilibrio entre sus facultades intelectuales y sus propensiones animales, aparentemente se había destruido". El caso de Phineas Gage llevó a pensar en la posibilidad de modificar la conducta produciendo lesiones cerebrales artificialmente (17). En 1892, Gottlieb Burkhardt extirpó áreas corticales anteriores de seis psicóticos agresivos; aunque dos de ellos murieron, los otros cuatro presentaron una disminución de la agresividad. En 1935, Carlyle Jacobson y John Fulton informaron que la extirpación de la corteza frontal en monos los volvía mucho más tranquilos, sin alterar otras funciones mentales (18).

En 1935 los neurólogos Egas Moniz y Almeida Lima desarrollaron la técnica denominada lobotomía prefrontal, que consistía en interrumpir las conexiones de la sustancia blanca de los lóbulos frontales con el sistema límbico, utilizando un artefacto llamado "leucótomo" (por lo cual Moniz ganó el Premio Nobel de Medicina en 1949. El segundo y último para un tratamiento psiquiátrico). En 1945 el neurólogo Walter J. Freeman y el cirujano James W. Watts modificaron la técnica de Moniz y Lima, introduciendo un leucótomo similar a un picahielo a través de la órbita, hasta llegar al cerebro; crearon así la leucotomía transorbitaria (la "lobotomía picahielo"), de rápida ejecución y con anestesia local, de modo que "podía llevarse a cabo en el mismo consultorio". El método se popularizó rápidamente y fue aplicado a psicóticos agresivos, depresivos severos y obsesivos-compulsivos que no respondían a las otras terapias existentes (principalmente insulinoterapia y electrochoque) (19). Durante las décadas de 1940 y 1950 más de 50 mil personas en todo el mundo fueron sujetas a la lobotomía; un ejemplar del New York Times decía que los cirujanos de aquel entonces "no dudaban en hacer operaciones del cerebro más de lo que dudarían para quitar un apéndice" (7). En sus momentos de auge, la lobotomía se llegó a usar en niños y adolescentes rebeldes, y hasta en disidentes políticos; "yo recuerdo tener unos ojos negros grandes e hinchados y quedarme en el hospital durante unos días porque al parecer tenía una infección", rememora Howard Dully, lobotomizado por el mismo Freeman a los 12 años (20). Según Sabbatini "la lobotomía prefrontal producía 'zombies', personas sin emociones, apáticas para todo, y con escasa iniciativa. Habían perdido además importantes funciones mentales, tales como una conducta social adecuada y la capacidad para planear acciones" (19). En 1947, el proyecto Columbia-Greystone, en los Estados Unidos, no encontró evidencia a favor de la efectividad del método. La aparición de los antipsicóticos contribuyó aún más al ocaso de la lobotomía, hasta que prácticamente desapareció del mapa. El fantasma de la misma continuó sin embargo en el imaginario popular (los mismos psicofármacos serían calificados como "lobotomía química" por sus detractores del movimiento antipsiquiátrico), extendiendo aún más el estigma no solo sobre el enfermo mental sino también sobre la práctica psiquiátrica. 


\section{LOS HOSPITALES-PRISIONES SOVIÉTICOS}

Durante las décadas de 1970 y 1980, cientos o quizás miles de disidentes políticos de la Unión Soviética fueron confinados en hospitales psiquiátricos. El marco teórico apropiado lo dio el psiquiatra Andréi Snezhnevski y su definición de "esquizofrenia lenta", como un trastorno de límites bastante laxos entre cuyos criterios figuraban: "originalidad, temor y desconfianza, formulaciones ideológicas peculiares, religiosidad, (...) apasionamiento, (...) mala adaptación al ambiente social, (...) reformismo" (21). De este modo, por el solo hecho de expresar una opinión abiertamente contraria al gobierno, cualquiera podía ser sometido a una "evaluación psiquiátrica" y confinado en un hospital. Aquellos considerados como especialmente peligrosos tenían como destino un hospital de máxima seguridad, a cargo del Ministerio del Interior Ruso. Además del encierro, los maltratos físicos, la mala alimentación, la falta de higiene y la compañía de enfermos mentales agresivos, los disidentes políticos eran sometidos a altas dosis de neurolépticos, sesiones frecuentes de electrochoque e insulinoterapia, y además al uso de atropina y sulfazina $(22,23)$.Muchas de estas personas desarrollaron síntomas de estrés postraumático, y su salida de la reclusión estuvo marcada por el estigma, la soledad, la desesperanza y -evidentemente- la desconfianza hacia cualquier tipo de ayuda médica psiquiátrica (22).

La explicación a los hospitales-prisiones soviéticos probablemente vaya más allá de una burda intromisión estatal en los fueros de la psiquiatría, y comprenda una ideologización extrema de la especialidad (y de la sociedad en general), que lleva a considerar como obviamente patológico el oponer resistencia al "pensamiento correcto" del régimen, inculcado como dogma incuestionable desde temprana infancia. $Y$ en esto la ideología política puede terminar asemejándose al fanatismo religioso. Tal es el peligro no solo de la dependencia ideológica de la psiquiatría, sino también de la laxitud y subjetividad en sus conceptos, sumada a una actitud paternalista y al uso del tratamiento como ejercicio de poder sobre los demás.

\section{Correspondencia}

Santiago Stucchi-Portocarrero

Correo electrónico: stucchi@amauta.rcp.net.pe

\section{REFERENCIAS BIBLIOGRÁFICAS}

1. Deuteronomio 28: 27-28. En: Santa Biblia. Antiguo y Nuevo Testamento. Gran Bretaña: Sociedades
Bíblicas en América Latina, 1960.p. 204.

2. Platón. Fedro o de la belleza. Versión en español de Patricio de Azcárate; Madrid: Medina y Navarro; 1871-1872.p.261-349. (Citado en octubre del 2013) URL disponible en: http://www.filosofia.org/cla/pla/ azc02261.htm.

3. Ackerknecht EH. Breve historia de la psiquiatría. Buenos Aires: Editorial Universitaria de Buenos Aires; 1962.

4. Callejo J. Breve historia de la brujería. Madrid: Ediciones Nowtilus SL; 2008.

5. Alexander FG, Selesnick ST. Historia de la psiquiatría. Una evaluación del pensamiento psiquiátrico desde los tiempos prehistóricos hasta nuestros días. Barcelona: Editorial Espaxs; 1966.

6. Kramer H, Sprenger J. El martillo de las brujas (MalleusMaleficarum)(Facsímil de la edición de 1874). Valladolid: Maxtor; 2004.

7. Porter R. Breve historia de la locura. Madrid: Turner Publicaciones SL; 2002.

8. Foucault M. Historia de la locura en la época clásica. México: Fondo de Cultura Económica; 1998.

9. Valdivia-Ponce O. Panorama de la psiquiatría en el Perú. Volumen 1. Lima: Universidad Nacional Mayor de San Marcos; 1991.

10. Von Tschudi JJ. El Perú. Esbozos de viajes realizados entre 1838 y 1842. Lima: Fondo Editorial de la Pontificia Universidad Católica del Perú; 2003.

11. Ruiz-Zevallos A. Medicina mental y modernización: Lima, 1850-1900. En: Panfichi A, Portocarrero F (ed). Mundos interiores: 1850-1950. Lima: Centro de Investigación de la Universidad del Pacífico; 2004.p. 373-396.

12. Debay A. Historia natural del hombre y de la mujer. Desde su aparición sobre el globo terrestre hasta nuestros días. Barcelona: Jané Hermanos Editores; 1892.

13. Francotte X. L'Anthropologie criminelle. Paris: Libraire J.B. BaillièreetFils; 1891.

14. Von Krafft-Ebing R. Psychopatia sexualis. New York: Special Books Inc; 1965.

15. Galton F. Eugenics: Its definition, scope, and aims. The American Journal of Sociology 1904; 10: 1.

16. Kühl S. The Nazi Connection: Eugenics, American Racism, and German National Socialism. Oxford: Oxford University Press; 2001.

17. Sabbatini RME. The amazing case of Phineas Gage. Brain \& Mind. $\mathrm{N}^{\circ}$ 2; 1997. (Citado en octubre del 2013) Disponible en: http://www.cerebromente.org. br/n02/historia/phineas.htm.

18. Grebb JA. Psicocirugía. En: Kaplan HI, Sadock BJ. Tratado de Psiquiatría. Sexta edición. Buenos Aires: Inter-Médica; 1997.p. 2060-4.

19. Sabbatini RME. The history of psychosurgery. Brain \& Mind. $N^{\circ} 2$; 1997. (Citado en octubre del 2013) Disponible en: http://www.cerebromente.org. 
Estigma, discriminación y concepto de enfermedad mental.

br/n02/historia/psicocirg_i.htm .

20. Gajilan AC. Survivor recounts lobotomy at age 12 . Procedure once considered legitimate medical treatment. CNN. November 30, 2005.(Citado en octubre del 2013). Disponible en: http://www.cnn. com/2005/HEALTH/conditions/11/30/pdg. lobotomy/index.html.

21. Reich W. El diagnóstico psiquiátrico como problema ético. En: Bloch S, Chodoff P, Green SA (eds). La ética en psiquiatría. Madrid: Triacastela, 2001: 189216.
22. Adler N, Gluzman S. Soviet Special Psychiatric Hospitals: Where the system was criminal and the inmates were sane. Br J Psychiatry 1993; 163: 713720 .

23. Bonnie RJ. Political abuse of psychiatry in the Soviet Union and in China: Complexities and controversies. J Am AcadPsychiatryLaw 2002; 30: 136-144.

Recibido: 20/10/2013

Aceptado: 25/11/2013 